\title{
Tight-binding description of impurity states in semiconductors
}

\section{F. Domínguez-Adame}

GISC, Departamento de Física de Materiales, Universidad Complutense, E-28040

Madrid, Spain

E-mail: adame@fis.ucm.es

\begin{abstract}
.
Introductory textbooks in solid state physics usually present the hydrogenic impurity model to calculate the energy of carriers bound to donors or acceptors in semiconductors. This model treats the pure semiconductor as a homogeneous medium and the impurity is represented as a fixed point charge. This model is only valid for shallow impurities and it also departs from the conventional view in solid state physics, where carriers move in a crystal lattice. As an alternative description of impurities in semiconductors, we present a minimal one-dimensional lattice model within the tight-binding approximation. The lattice model is valid for deep and shallow impurities. In the latter case, the results are in agreement with the predictions of the hydrogenic impurity model. The underlying ideas are simple and knowledge of advanced quantum mechanics is not required. Thus, this alternative model could be suitable for introductory courses in solid state physics and materials science.
\end{abstract}

PACS numbers: 71.55.-i, 71.55.Cn, 71.15.Ap

European Journal of Physics 33, 1083 (2012) 


\section{Introduction}

Since the invention of the first solid state transistor by Shockley, Bardeen and Brattain at Bell Laboratories, which garnered the 1956 Nobel Prize in Physics, semiconductors are the basis of most modern electronic devices. Semiconductors are materials of choice for many applications because their electronic and optical properties can be tailored by adding small amounts of foreign atoms during the fabrication process, referred to as impurities (see, e.g., Ref. [1]). Electron concentration can be increased by adding dopant elements known as donors ( $n$ doping). Similarly, an excess of holes is achieved by adding acceptors to the semiconductor ( $p$ doping). At zero temperature, any free carriers from donors and acceptors are bound to their impurity atoms due to an attractive Coulomb potential. On increasing temperature, impurities can be thermally ionized and free carriers are released to the conduction or valence bands. Usually impurities are classified as deep or shallow according to their ionization energy. Shallow impurities are defined as those impurities whose ionization energy is comparable or smaller than the thermal energy at room temperature. As an example of shallow impurity, the ionization

energy of a $\mathrm{P}$ atom in $\mathrm{Ge}$ is $12 \mathrm{meV}$, smaller than $25 \mathrm{meV}$, the thermal energy at room temperature [2]. On the contrary, S, Se and Te introduce deep donor levels and Zn, Cd and $\mathrm{Mn}$ introduce deep acceptor levels in Ge. In all cases the ionization energy is larger than $30 \mathrm{meV}[3]$.

Shallow impurities are usually known as hydrogenic impurities since they are well described by the hydrogen atom model. The underlying assumptions behind this model are that the binding energy is small compared with the energy gap and the spatial extent of the wave function is larger than the lattice period. As a consequence, carriers have an energy close to the band edge, move with an energy-independent effective mass $m^{*}$ and see the uniform medium of the semiconductor characterized by a dielectric constant $\epsilon$ that screens the Coulomb interaction with the impurity. Students of solid state or semiconductor physics have a background in quantum physics and are familiar with the Schrödinger picture of the hydrogen atom. For this reason, most introductory textbooks in these subjects rely on the hydrogenic impurity model to calculate the binding energy of the carrier and the spatial extent of the wave function in the ground state [2]. This model predicts that the ground-state binding energy is the effective Rydberg $\mathrm{Ry}^{*}=\left(m^{*} / m \epsilon^{2}\right) \times 13.6 \mathrm{eV}$ and the spatial extent is determined by the effective Bohr radius $a^{*}=\left(m \epsilon / m^{*}\right) \times 0.053 \mathrm{~nm}, m$ being the free electron mass [2]. As occurs in the hydrogen atom, both magnitudes are related since $\mathrm{Ry}^{*}=\hbar^{2} / 2 m^{*}\left(a^{*}\right)^{2}$. In a typical semiconductor, $m^{*}$ is smaller than $m$ and $\epsilon \sim 10$. Thus, Ry* is only few tenths of $\mathrm{meV}$ and $a^{*}$ is several nm, i.e., much larger than the lattice period. The large spatial extent of the carrier wave function allows to consider the semiconductor as a homogeneous medium. It is important to remark that the binding energy depends only on the semiconductor parameters $\left(m^{*}\right.$ and $\left.\epsilon\right)$ and becomes independent of the peculiarities of the impurity atom. In other words, the hydrogenic model predicts that all shallow impurities have the same ionization energy in a given semiconductor. 
The hydrogenic impurity model departs from the conventional view in solid state physics, where carriers move in the periodic potential that arises from the arrangement of atoms in a crystal structure. When the semiconductor is doped, the periodicity of the lattice is broken and, from a strict point of view, Bloch theorem no longer applies. As a consequence, the calculation of the ground-state energy of an electron (or a hole) bound to an impurity is a formidable task, even within the one-electron approximation, and it is beyond the scope of introductory textbooks.

The aim of this work is to fill the gap between the hydrogenic impurity model (effective homogeneous medium with a fixed point charge) and more elaborated descriptions of impurity states that take into account the crystalline nature of semiconductors. The goal is to present a one-dimensional lattice model that could be suitable for introductory courses in solid state and semiconductor physics. The model will be discussed at two stages of complexity. After introducing the lattice model, which is a simplified version of the celebrated Anderson impurity model [4], in a first stage the solution is obtained in a rather heuristic way, advancing the form of the wave function and supporting the trial solution by analogy with a $S$ orbital of the hydrogen atom. This part of the presentation is self-contained and little further reading is needed. For advanced students, the exact solution of the lattice model will be discussed in a second stage. In this case, a basic knowledge of Green functions for tight-binding Hamiltonians is required and the reader will be referred to the well-known textbook by Economou for additional information [5].

\section{Impurity lattice model}

Single impurity atoms in semiconductor crystals can be incorporated on lattice sites (substitutional impurities) or interstitial sites (interstitial impurities). For the sake of concreteness, only interstitial impurities in a one-dimensional lattice will be considered hereafter, as shown schematically in Fig. 1(a). Electron states will be described within the tight-binding framework with one orbital per site (see Ref. [6] for a brief introduction to this approach in nonperiodic lattices). The impurity is attached at site $n=0$ of an otherwise periodic lattice. The overlap integral between this site and the impurity is denoted as $-J_{I}$, and $-J$ is the overlap integral between nearest-neighbour sites. In addition, the site energy is set to zero to avoid the profusion of free parameters.

Let $\psi_{n}$ with $n=0, \pm 1, \pm 2, \ldots$ and $\psi_{I}$ be the wave function amplitudes at lattice and impurity sites, respectively. The equation for the amplitude at the impurity site is $E \psi_{I}=-J_{I} \psi_{0}$, where $E$ is the particle energy. Taking into account the equation for the amplitudes at the lattice sites $E \psi_{n}=-J\left(\psi_{n+1}+\psi_{n-1}\right)-J_{I} \delta_{n 0} \psi_{I}$ one gets

$$
\left[E-\varepsilon(E) \delta_{n 0}\right] \psi_{n}=-J\left(\psi_{n+1}+\psi_{n-1}\right),
$$

where $\varepsilon(E)=J_{I}^{2} / E$. Therefore, we can see that the original lattice with an interstitial impurity is equivalent to a lattice with a substitutional impurity at the origin, where the site energy $\varepsilon(E)$ depends on the particle energy, as depicted schematically in Fig. 1(b). 
a)

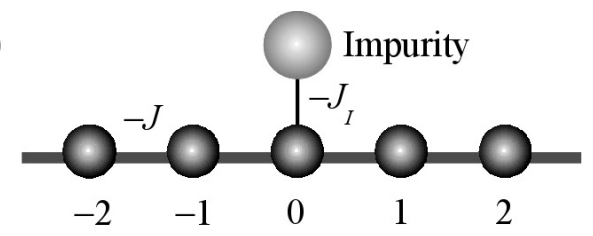

b)

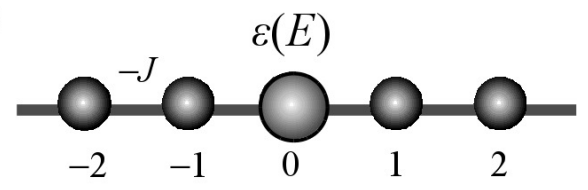

Figure 1. a) Schematic view of an interstitial impurity attached to a one-dimensional lattice. b) Equivalent substitutional impurity with energy-dependent site energy $\varepsilon(E)=J_{I}^{2} / E$.

The tight-binding equation (1) can be easily solved when the interstitial impurity is removed, namely $J_{I}=0$ and $\varepsilon(E)=0$. In this limiting case the lattice is periodic and we can make use of the Bloch theorem to write the unnormalized solution of Eq. (1) as $\psi_{n}^{0}=\exp (i k a n)$, where $a$ is the lattice spacing and the superscript refers to the homogeneous lattice case $\left(J_{I}=0\right)$. The corresponding energy values form a band of allowed states $E_{k}=-2 J \cos (k a)$. The minimum of the band is located at $k=0$ when $J>0$ and the corresponding energy is $E_{\min }=-2 J$. Thus, the case $J>0$ is suitable to study impurity states below the lower edge of the conduction band (donor states). Acceptor states above the maximum of the valence band can be described in a similar fashion reversing the sign of the overlap integral $J$. For definiteness we restrict ourselves to $J>0$ hereafter. The effective mass of the electron close to the lower band edge is given by

$$
m^{*}=\hbar^{2}\left(\frac{\partial^{2} E_{k}}{\partial k^{2}}\right)_{k=0}^{-1}=\frac{\hbar^{2}}{2 J a^{2}} .
$$

The Bloch solution with $k$ real is valid for the homogeneous lattice and represents a traveling wave defined at the lattice sites. When the impurity is attached to the lattice, the translational symmetry is broken and the Bloch theorem with $k$ real is not longer valid. Nevertheless, we can obtain a localized in space solution replacing $k$ in the Bloch solution by an imaginary parameter. In order to obtain a normalizable solution we make the substitution $k \rightarrow i \lambda / a(k \rightarrow-i \lambda / a)$ in the right (left) half of the lattice. Experienced readers will notice that this is exactly the same replacement used to describe midgap Tamm states arising at the surface of wide gap semiconductors. Thus, in order to solve Eq. (1) we propose the trial solution $\psi_{n}=\exp (-\lambda|n|)$ with $\lambda>0$. Notice that this is nothing but the one-dimensional version of a $S$ state in the hydrogen model, decaying exponentially with distance. Therefore, we can define an effective Bohr radius as $a^{*}=a / \lambda$. We will come back to this point later.

Inserting the ansatz $\psi_{n}=\exp (-\lambda|n|)$ in Eq. (1) for $n=0$ leads to $E\left(E+2 J e^{-\lambda}\right)=$ $J_{I}^{2}$, and when $n \neq 0$ the result is $E=-2 J \cosh \lambda$. In this way we have two algebraic 
equations for the two unknowns, $E$ and $\lambda$. Combining both equations we arrive at

$$
\sinh (2 \lambda)=\frac{J_{I}^{2}}{2 J^{2}} .
$$

The right-hand side of equation (3) is positive, implying that $\lambda$ is positive, in agreement with our previous assumption. Once $\lambda$ is known, we can obtain the energy level from $E=-2 J \cosh \lambda$. Since $\cosh \lambda>1$, the resulting localized level lies below the lower band edge $\left(E<E_{\min }=-2 J\right)$, as occurs in the hydrogenic model too.

It is important to stress that these results apply to both deep and shallow impurities since no constraints are imposed on the binding energy. Nevertheless, particularly simple expressions arise by considering shallow impurities. In this case the binding energy is small $\left(E \lesssim E_{\min }=-2 J\right)$ and the effective Bohr radius is much larger than the lattice spacing $\left(a^{*}=a / \lambda \gg a\right)$. Consequently $\lambda \ll 1$ and Eq. (3) reduces to $\lambda \simeq J_{I}^{2} / 4 J^{2}$. The effective Bohr radius becomes

$$
a^{*}=\frac{4 J^{2}}{J_{I}^{2}} a .
$$

Therefore, the limit of a shallow impurity is achieved when the foreign atom is only weakly coupled to the host lattice, namely $J_{I} \ll J$. In this limit we obtain $E \simeq-2 J-J_{I}^{4} / 16 J^{3}=E_{\min }-J_{I}^{4} / 16 J^{3}$ and the effective Rydberg then is

$$
\mathrm{Ry}^{*}=E_{\min }-E=\frac{J_{I}^{4}}{16 J^{3}} .
$$

As a test of the consistency of the model, we can use the general relationship obtained within the hydrogenic impurity model $\mathrm{Ry}^{*}=\hbar^{2} / 2 m^{*}\left(a^{*}\right)^{2}$ to calculate the effective mass as $m^{*}=\hbar^{2} / 2 \mathrm{Ry}^{*}\left(a^{*}\right)^{2}$. Using Eqs. (4) we obtain exactly the same effective mass given by $(2)$.

Before going further into the exact solution of the lattice model, let us compare the theoretical predictions with the experimental values available in the literature. To this end, we consider GaAs, one of the most studied semiconductors. The conduction band is isotropic around the center of the Brillouin zone ( $\Gamma$ valley) and the effective mass is $m^{*}=0.067 m$. Taking into account that the dielectric constant is $\epsilon=12.9$, the hydrogenic model predicts $\mathrm{Ry}^{*}=5.5 \mathrm{meV}$. The calculated value agrees remarkably well with the experimental binding energy of some donors in GaAs, such as $\mathrm{S}$, Se, Si, Ge and $\mathrm{Sn}$, for which the admitted value is around $5.8 \mathrm{meV}$ [7]. As we commented above, this limit is also captured by the lattice model. However, when the donor is Te, the binding energy is $30 \mathrm{meV}$, much larger than that predicted by the hydrogenic model. Fortunately, the lattice model has three adjustable parameters $\left(J, J_{I}\right.$ and $\left.a\right)$ but there are only two parameters in the hydrogenic model $\left(m^{*}\right.$ and $\left.\epsilon\right)$. This means that we could select a larger value of $J_{I}$ (roughly 1.5 times larger than the previous one) and, according to Eq. (4b), the binding energy increases. It is worth mentioning that the change of $J_{I}$ will not affect the effective mass (2), which should be constant for the same semiconductor. 


\section{Exact solution}

In the previous section it was pointed out that the one-dimensional lattice with an interstitial impurity is equivalent to a one-dimensional lattice with an energy-dependent site energy $\varepsilon(E)$ in one of the nodes (see Fig. 1). The topology of the latter is strictly onedimensional, in the sense that all sites have only two nearest-neighbour sites. Solution of the tight-binding Hamiltonian for an electron in a one-dimensional lattice with a single energy-independent site energy is presented in detail in Ref. [5]. Here we will apply these results to the problem at hand. For brevity we will skip the details of the calculations and restrict ourselves to the peculiarities of having a node of the lattice with an energy-dependent site energy $\varepsilon(E)$.

The equation for the amplitudes $\psi_{n}$ given by (1) can be obtained from the tightbinding Hamiltonian $\widehat{H}=\widehat{H}_{0}+\widehat{H}_{I}$, where $\widehat{H}_{0}$ corresponds to the periodic lattice

$$
\widehat{H}_{0}=-J \sum_{n}[|n\rangle\langle n+1|+| n+1\rangle\langle n|],
$$

and the perturbation due to the impurity at the origin is

$$
\widehat{H}_{I}=|0\rangle \varepsilon(E)\langle 0| \text {. }
$$

$|n\rangle$ is the localized orbital at site $n$ and the electron wave function is $|\psi\rangle=\sum_{n} \psi_{n}|n\rangle$. According to Ref. [5], the energy of the bound state lying outside the band is given by the pole of the Green function $\widehat{G}(E)=(E-\widehat{H}+i \eta)^{-1}, \eta$ being a small positive quantity. The Green function $\widehat{G}(E)$ can be expressed in terms of $\widehat{G}_{0}(E)=\left(E-\widehat{H}_{0}+i \eta\right)^{-1}$ as

$$
\widehat{G}(E)=\widehat{G}_{0}(E)+\widehat{G}_{0}(E)|0\rangle \frac{\varepsilon(E)}{1-\varepsilon(E) G_{0}(0,0 ; E)}\langle 0| \widehat{G}_{0}(E)
$$

where $G_{0}\left(\ell, \ell^{\prime} ; E\right)=\left\langle\ell\left|\widehat{G}_{0}\right| \ell^{\prime}\right\rangle$. The pole is then determined from the condition

$$
G_{0}(0,0 ; E)=\frac{1}{\varepsilon(E)}=-\frac{E}{J_{I}^{2}} .
$$

Outside the band $\left(E^{2}>4 J^{2}\right)$, the diagonal matrix element of the Green function is [5]

$$
G_{0}(0,0 ; E)=\frac{1}{\sqrt{E^{2}-4 J^{2}}} .
$$

Notice that (7) implies that the energy is real and negative. It is a matter of simple algebra to obtain that $E=-2 J \cosh \lambda$, using (3) as a definition of $\lambda$.

The spatial extent of the bound state can be established from the exponential decrease of the Green function with distance $\psi_{n} \sim G_{0}(n, 0 ; E) \sim \exp (-\lambda|n|)$, where [5]

$$
\lambda=-\ln \left(\frac{|E|}{2 J}-\sqrt{\frac{E^{2}}{4 J^{2}}-1}\right) .
$$

It is straightforward to check that (8) agrees with (3) when $E=-2 J \cosh \lambda$. 


\section{Conclusions}

In conclusion, in this paper we have presented a one-dimensional lattice model of an interstitial impurity in a semiconductor. Using a tight-binding approach, we found that the interstitial impurity is equivalent to a substitutional impurity with an energydependent site energy. We have obtained the energy of the carrier and the spatial extent of the wave function for both deep and shallow impurities. In the latter case, we have identified the binding energy in the ground state and the extent of the wave function with the effective Rydberg $\mathrm{Ry}^{*}$ and Bohr radius $a^{*}$, respectively. From the dispersion relation we have also calculated the effective mass $m^{*}$ at the bottom of the band. The relationship between these magnitudes is the same as that found in the well-known hydrogenic impurity model. We claim that the level of complexity of both hydrogenic impurity and lattice model are similar, and the latter could also be presented in introductory courses in solid state physics or materials science. Finally, we have also

found the exact solution using the Green function formalism, which could be suitable to introduce the problem in advanced courses.

The author thanks J. Munárriz and C. González-Santander for a critical reading of the manuscript. This work was supported by MICINN (Project MAT2010-17180).

\section{References}

[1] Seeger K 1991 Semiconductor Physics. An Introduction (Berlin: Springer-Verlag)

[2] Ashcroft N W and Mermin N D 1976 Solid State Physics (New York: Saunders)

[3] Tyler W W 1959 J. Phys. Chem. Solids 859

[4] Anderson P W 1961 Phys. Rev. 12441

[5] Economou E N 2006 Green's Functions in Quantum Physics (Berlin: Springer-Verlag)

[6] Domínguez-Adame F and Malyshev V A 2004 Am. J. Phys. 72226

[7] Yu P Y and Cardona M 2005 Fundamentals of Semiconductors. Physics and Materials Properties (Berlin: Springer-Verlag) p. 169 